\title{
Dysfonction immunitaire induite par la ventilation mécanique
}

\section{Ventilator-induced immune dysfunction}

\author{
J.M. Tadié $\cdot$ A. Gacouin $\cdot$ Y. Le Tulzo \\ Reçu le 2 décembre 2013 ; accepté le 26 décembre 2013 \\ (C) SRLF et Springer-Verlag France 2014
}

Résumé La ventilation mécanique (VM), même si elle fait partie de l'arsenal thérapeutique indispensable en réanimation, va induire ou aggraver des lésions pulmonaires. Les cellules du parenchyme pulmonaire ainsi que les cellules immunitaires du poumon vont être capables de convertir les forces mécaniques générées par la VM en un signal biologique qui va entraîner une réaction inflammatoire. Cette inflammation « stérile » locale puis systémique va entraîner une dysfonction du système immunitaire, source de morbidité et de mortalité pour le patient de réanimation.

Mots clés Ventilation mécanique $\cdot$ Dysfonction immunitaire · SDRA

\begin{abstract}
Although mechanical ventilation is an essential support in patients admitted to the intensive care unit, clinical and experimental studies have shown that it could be harmful and could induce lung injury. Pulmonary and immune cells can convert mechanical stimuli into biological signals that will lead to inflammation. This sterile inflammation both locally and systemically will cause immunosuppression.
\end{abstract}

Keywords Mechanical ventilation · Immune dysfunction · ARDS

\section{Introduction}

Le parenchyme pulmonaire représente une surface d'échange extrêmement large, en contact avec l'environnement extérieur. Par conséquent, le système immunitaire du poumon doit faire face à des situations disparates. Lors d'une atteinte respiratoire, neurologique ou encore lors

J.M. Tadié $(\bowtie) \cdot$ A. Gacouin $\cdot$ Y. Le Tulzo

Réanimation médicale, service des maladies infectieuses

et réanimation médicale, équipe infectiologie CIC-Inserm-0203,

hôpital Pontchaillou, CHU Rennes, 2 rue Henri Le Guillloux,

F-35033 Rennes cedex

e-mail : jean-marc.tadie@chu-rennes.fr d'une chirurgie sous anesthésie générale, le poumon va être exposé aux contraintes engendrées par la ventilation mécanique (VM). Il est désormais acquis que la VM peut générer et/ou aggraver des lésions pulmonaires [1]. La ventilation en pression positive déclenche une réponse biologique, initialement limitée au poumon, puis une réponse inflammatoire systémique dont une des conséquences pourrait être une dysfonction immunitaire plus «globale » $[2,3]$. Les patients de réanimation ventilés ont, pour la très grande majorité d'entre eux, une fonction pulmonaire altérée et une dysfonction immunitaire du fait de la pathologie initiale. En outre, la limitation de la toux secondaire à la présence d'une prothèse trachéale et l'altération de la clairance mucociliaire, de la composition du surfactant, des capacités de phagocytose et les mécanismes de défense des cellules épithéliales sont à l'origine d'une importante diminution des défenses antiinfectieuses du poumon. Le mécanisme physiopathologique serait comparable à la dysfonction immunitaire observée lors du choc septique [4] : l'interaction entre une « alarmine » et le " pattern recognition receptor» (" récepteur reconnaissant des formes ») porté par une cellule immunitaire ou parenchymateuse pulmonaire, induirait une majoration de mécanismes pro- et anti-inflammatoires responsables d'une immunodépression. Cette dysfonction immunitaire induite ou aggravée par la VM serait un facteur de surmorbidité et de surmortalité en réanimation [5].

\section{Ventilation mécanique et effet sur le système immunitaire : biotraumatisme}

\section{Ventilator induced lung injury / ventilator associated lung injury}

Il faut différencier deux mécanismes lorsque l'on considère les lésions pulmonaires liées à la ventilation mécanique (VM) [6] :

- les lésions pulmonaires induites par la VM sur poumon sain. Elles ont essentiellement été étudiées dans le cadre 
d'études utilisant les modèles animaux. On parle dès lors de Ventilator Induced Lung Injury ou VILI. La cause principale est un volume courant (Vt) trop élevé $(>12 \mathrm{~mL} / \mathrm{kg}$ de poids idéal) ;

- les lésions pulmonaires majorées par la VM, chez des patients hospitalisés en réanimation et présentant déjà un syndrome de détresse respiratoire aiguë (SDRA). On parle dès lors de Ventilator Associated Lung Injury ou VALI.

Dans la suite de cette revue, nous ne différencierons pas ces deux mécanismes et nous utiliserons le terme de VILI.

Presque tous les patients admis en réanimation présentent deux particularités importantes : des lésions pulmonaires plus ou moins importantes et une immunodépression plus ou moins profonde. Ainsi, ces patients vont développer, en plus, des lésions pulmonaires secondaires, majorées par la VM. Ces lésions vont générer une réponse inflammatoire à l'origine d'une dysfonction du système immunitaire. Il faut rappeler que la pathologie responsable de l'hospitalisation en réanimation ainsi que les antécédents du patient et les médicaments administrés ont aussi une part importante dans tous ces processus.

Ces lésions de VILI sont essentiellement de trois types :

- lésions de barotraumatisme, synonymes de fuite d'air extra-alvéolaire, du fait généralement d'une rupture alvéolaire. Ce barotraumatisme survient généralement chez les patients présentant la forme la plus sévère de SDRA. L'altération des propriétés élastiques du poumon et la réduction de son volume aéré induisent alors une forte élévation des pressions pour un faible volume insufflé dans les alvéoles. La surdistension des alvéoles induite par la ventilation peut provoquer une rupture alvéolaire. Le pneumothorax en est l'expression la plus typique avec le pneumomédiastin. Le barotraumatisme est favorisé à la fois par la pathologie pulmonaire sous-jacente et par l'utilisation de pressions excessives délivrées par le ventilateur. L'incidence du barotraumatisme au cours du SDRA ne dépasse pas $10 \%$ depuis la réduction du volume courant et la limitation de la pression de plateau. Ce type de lésion n'entre classiquement pas dans le cadre de la dysfonction immunitaire induite par la VM $[7,8]$;

- lésions de volotraumatisme. L'hétérogénéité du parenchyme pulmonaire réduit considérablement le volume pulmonaire normal (concept du baby lung) [9]. Lors de la VM, les zones de parenchyme pulmonaire non ou peu atteintes vont être exposées à des forces mécaniques inhabituelles alors que les alvéoles atteintes resteront collabées : il existe alors un risque de surdistension de ces zones saines générant des lésions de l'alvéole et de la membrane alvéolo-capillaire. De plus, cette inhomogénéité va entraîner un collapsus intermittent des alvéoles atteintes mais aussi d'alvéoles saines. Ceci va entraîner des lésions dites d'atelectrauma à l'origine d'une majoration des lésions de la barrière alvéolo-capillaire [10] ;

- lésions de biotraumatisme. À la différence des lésions précédemment décrites, le concept de biotraumatisme ne repose pas sur des modifications macroscopiques de structures du parenchyme pulmonaire. Les forces biophysiques générées par la VM, l'étirement cyclique (cyclic stretch) sur l'alvéole et les cellules du poumon ont pour conséquence une altération de la physiologie normale des différentes cellules du poumon, à l'origine d'une réponse inflammatoire locale puis systémique, source de modifications de l'immunité [6].

\section{Biotraumatisme et modulation de la réponse immunitaire}

Le biotraumatisme est donc un phénomène biologique secondaire au phénomène de VILI. Il survient essentiellement lorsque le poumon est lésé (SDRA) et ventilé, même si des données de plus en plus nombreuses suggèrent l'existence d'un VILI sur poumon sain, avec des durées de VM courte (voir paragraphe IV-B) $[2,11,12]$.

\section{Mécanotransduction}

Toutes les cellules du parenchyme pulmonaire (fibroblastes, macrophages, polynucléaires neutrophiles [PNN] et éosinophiles, pneumocytes, cellules épithéliales bronchiques...) vont être sensibles aux forces générées par la $\mathrm{VM}$ et contribuer aux mécanismes du VILI et à la dysfonction immunitaire induite par la VM [13,14]. En effet, comme les structures osseuse ou vasculaire, les cellules pulmonaires peuvent modifier leur structure, leur métabolisme intracellulaire en fonction de leur environnement mécanique grâce à des mécanorécepteurs. Il existe différents types de forces : force statique ou cyclique, uni- ou multidirectionnelle, uniaxiale ou biaxiale. Leur description échappe à la thématique de cette revue, cependant, même si les modèles expérimentaux différencient précisément les forces étudiées sur les différentes populations cellulaires, il semble que les forces mécaniques impliquées dans le VILI soient de tous les types (pression transpulmonaire augmentée, stress de déformation, étirement) et touchent les différentes cellules du poumon. Notons que même si ces forces sont transmises à l'ensemble du parenchyme pulmonaire, la VM va surtout générer des lésions cellulaires dans les zones de poumon lésées, plus sensibles à la surdistention et aux phénomènes d'atelectrauma.

Les mécanismes impliqués dans la transformation d'un message physique (= force appliquée à la cellule) en un message ou signal biologique est appelé mécanotransduction, dont tous les mécanismes ne sont pas élucidés. Cependant, 
trois mécanismes importants semblent être impliqués dans la survenue du VILI $[2,14]$ :

- effets sur les canaux ioniques sensibles à la déformation. Ce sont essentiellement des canaux ioniques calciques qui peuvent être activés ou inhibés en fonction du signal ;

- modifications des enzymes membranaires. Les déformations de la membrane cellulaire activent des enzymes membranaires (tyrosine kinase et phospholipase C- $\gamma$ ) générant un signal intracellulaire ;

- transmission directe au milieu intracellulaire d'un stress physique extérieur par le cytosquelette et la matrice extracellulaire (MEC).

Ces modifications vont induire des transformations à différents niveaux :

- cellulaire : les réactions cellulaires au stress mécanique vont être diverses et toutes ne sont probablement pas décrites : production d'espèces réactives de l'oxygène, acidification du milieu extracellulaire, accélération de phénomènes apoptotiques [15-17]. De plus, les modifications du milieu extracellulaire ainsi que la sécrétion de facteurs chimiotactiques vont entraîner un afflux de PNN, responsables d'une majoration des lésions en produisant différentes molécules (cytokines, chimiokines, Neutrophil extracellular traps, espèces réactives de l'oxygène, élastase...) [6]. On assiste alors à une modification de cellularité de l'alvéole, avec généralement la présence d'une alvéolite à PNN associée à un liquide de lavage broncho-alvéolaire (LBA) riche en cytokines. Ces modifications peuvent survenir même lors de VM de courte durée sur poumon sain. En effet, l'analyse de LBA chez des patients sous VM pour une procédure chirurgicale, montre une cellularité augmentée et des concentrations de cytokines dans le lavage broncho-alvéolaire [interleukine (IL)-6 et Tumor necrosis factor (TNF)- $\alpha$ ] plus élevées que chez les sujets contrôles [18,19]. Si les PNN jouent un rôle prépondérant dans la physiopathologie du VILI et de la dysfonction immunitaire associée, les macrophages alvéolaires activés par la VM jouent aussi un rôle important dans l'inflammation [20] ;

- moléculaire : la production de molécules par les cellules inflammatoires (essentiellement PNN et macrophages) ainsi que par les cellules du parenchyme pulmonaire seront à l'origine d'une majoration des lésions pulmonaires, soit directement (neutrophils extracellular traps [NET] et espèces réactives de l'oxygène [ROS]), soit indirectement par le recrutement ou l'action sur d'autres cellules (interaction alarmine-récepteur spécifique) $[3,6,11,12,14]$. Ce sont les interactions entre ces molécules et ces cellules, ainsi que leur diffusion systémique qui entraîneront une immunodépression induites par la VM. Cette partie sera développée dans le paragraphe suivant;
- de nombreuses études ont démontré que la VM modifiait l'expression de certains gènes pro-inflammatoires. Pugin et al. ont démontré que l'étirement cyclique des alvéoles entraînait une activation de gènes pro-inflammatoire et la sécrétion des médiateurs par les macrophages alvéolaires. Cette traduction transcriptionnelle des forces générées par la VM est présente dans la plupart des cellules du poumon [20]. De plus, Copland et al. ont montré en utilisant un modèle de VM chez le rat, qu'après 30 minutes de VM avec un haut $\mathrm{Vt}$, des modifications génétiques (expression ou inhibition) pouvaient être mise en évidence, bien avant l'apparition des lésions anatomiques [21].

Ainsi la ventilation mécanique va générer des lésions mécaniques qui auront une traduction biologique : le biotraumatisme. Ce biotraumatisme engendre de nombreuses réactions qui vont entraîner une dysfonction immunitaire locale, intrapulmonaire, mais aussi systémique.

\section{Réponse immunitaire du poumon ventilé et lésé}

Nous ne détaillerons pas ici les caractéristiques propres du système immunitaire du poumon car les acteurs impliqués sont essentiellement des cellules « classiques » du système immunitaire inné (polynucléaires neutrophiles et macrophages), même si le poumon présente quelques caractéristiques dont nous parlerons brièvement [22,23]. Le poumon est la plus grande surface épithéliale de l'organisme en contact avec l'environnement extérieur et est sensible aux variations de pressions et de forces exercées sur lui. Il a de plus la particularité d'être traversé par la totalité du débit sanguin de l'organisme. Et ainsi, tout processus inflammatoire local va avoir rapidement une répercussion systémique.

Le système immunitaire du poumon est composé d'un système de défense non spécifique ou inné, et d'un système spécifique. Le système immunitaire inné pulmonaire comporte la barrière épithéliale, le système mucociliaire trachéobronchique, des peptides antimicrobiens : lysozyme et protéine du surfactant. Les cellules du système inné sont les cellules phagocytaires (PNN, monocyte et macrophage), les cellules dendritiques, les cellules épithéliales, les basophiles, les cellules mastocytaires, les polynucléaires éosinophiles et les cellules NK [22].

Il est habituel de considérer la clairance mucociliaire comme la première ligne de défense. En effet, la très grande majorité des particules inhalées sont éliminées grâce au fonctionnement de l'appareil mucociliaire : soit les particules inhalées sont piégées par le mucus recouvrant le rhinopharynx, soit elles sont piégées par les voies respiratoires hautes et « remontées » par l'appareil ciliaire pour être déposées dans le pharynx puis dégluties. Cependant, la 
colonisation de l'arbre trachéobronchique à partir de la flore oropharyngée est un phénomène qui arrive très précocement chez les malades ventilés en réanimation. La présence d'une prothèse trachéale altère la clairance mucociliaire et gêne la toux [24].

La deuxième ligne de défense est constituée par le système immunitaire inné ; la troisième ligne par le système acquis que nous n'étudierons pas, car il ne semble pas impliqué dans cette dysfonction immunitaire induite par la VM.

Les cellules immunitaires du poumon jouant un rôle dans la dysfonction immunitaire induite par la VM sont :

- le macrophage : cette cellule est présente dans l'espace alvéolaire et aussi intraparenchymateux. Ses fonctions immunes reposent sur ses capacités de phagocytose et de bactéricidie. Elle peut produite et libérer de nombreuses molécules jouant un rôle important dans la réponse inflammatoire. Ses capacités de phagocytose des bactéries sont diminuées en VM, comme ses capacités d'efférocytose (phagocytose de cellules apoptotiques). De plus, lors de la VM, les macrophages alvéolaires présentent une hyporéactivité lors d'une exposition aux bactéries. Enfin, ils participent au recrutement des PNN en secrétant des cytokines $[6,20]$;

- le PNN est peu présent à l'état basal $(<1 \%$ des cellules dans un lavage broncho-alvéolaire normal), la plupart des PNN sont marginés le long des parois des microvaisseaux. Ses fonctions principales sont, comme le macrophage, phagocytose et bactéricidie. Cependant, cette cellule présente comme particularité d'être recrutable extrêmement rapidement $[22,23,25]$. Les PNN deviennent ainsi majoritaires dans l'alvéole lors du VILI, et pourraient être les cellules les plus impliquées dans la dysfonction immunitaire induite par la VM. La capacité des PNN à être recrutés pour produire des lésions pulmonaires semble être dépendante de facteurs chimiotactiques. En effet, le blocage du récepteur CXCR2 (récepteur de certaines chimiokines de la famille $\mathrm{CXC}$ ) ou son absence (souris délétées) entraînent une diminution des lésions secondaires au VILI [26]. Ces PNN jouent en plus un rôle important dans le maintien et/ou l'aggravation des phénomènes inflammatoires : il a été démontré que la clairance des PNN apoptotiques (efférocytose) était une étape fondamentale dans la résolution d'un épisode inflammatoire, et tout mécanisme diminuant l'efférocytose aggrave les lésions induites par l'inflammation [27]. De plus, les PNN peuvent majorer les lésions lors d'une stimulation inflammatoire en produisant des ROS ou des NETs. Il a été démontré que ces deux mécanismes de défense antibactériens pouvaient majorer les lésions parenchymateuses pulmonaires et perpétuer ainsi l'inflammation [28];

- des études récentes ont retrouvé un rôle important des cellules épithéliales dans la réponse immunitaire innée du poumon. En plus de leur fonction mucociliaire, ces cellules expriment différents récepteurs (dont Toll-like receptor 4 ou TLR4) et peuvent synthétiser de nombreuses cytokines ainsi que des facteurs chimiotactiques qui entretiendront l'inflammation [23] ;

- enfin, le surfactant joue un rôle très important dans le maintien de l'homéostasie pulmonaire. La VM peut induire une modification des concentrations en protéines et lipides du surfactant (augmentation de la lipopolysaccharid binding protein [LBP] et $\mathrm{sCD} 14$, diminution de surfactant protein [SP]-A et SP-D), entraînant ainsi une réactivité accrue du poumon aux phénomènes inflammatoires [22].

Schématiquement, la dysfonction immunitaire induite par la VM repose sur l'interaction entre une molécule et une cellule portant un récepteur. Les récepteurs impliqués appartiennent au groupe des Pattern recognition receptors (PRRs). Ces récepteurs reconnaissent les agents infectieux et sont à l'origine de l'initiation de la réponse immunitaire innée contre l'infection. Les PRRs sont exprimés par les cellules du système immunitaire et par les cellules parenchymateuses du poumon. Ces récepteurs peuvent se lier à différentes molécules microbiennes (appelées Pathogen-associated molecular patterns [PAMPs]) et ainsi déclencher la réaction de défense contre une infection donnée. Mais ce récepteur peut aussi reconnaître des molécules non infectieuses, inflammatoires, appelées alarmines ou Damage-associated molecular pattern (DAMPs). Cette liaison va entraîner la production de cytokines pro- et anti-inflammatoires, d'interféron, de facteurs chimiotactiques, ainsi que d'autres alarmines qui majoreront la réponse inflammatoire aboutissant à un véritable syndrome de compensation anti-inflammatoire (CARS ou compensatory anti-inflammatory response syndrome). Comme les PRRs, les alarmines sont secrétées par les cellules immunitaires (PNN et macrophages) ou par les cellules du parenchyme pulmonaire lésé $[22,29]$. La représentation schématique des mécanismes est représentée Figure 1.

La liaison PRRs-DAMPs va activer des voies de signalisation intracellulaire qui conduiront à une diminution des capacités de défense (bactéricidie, phagocytose ou efférocytose), ainsi qu'à la sécrétion d'autres cytokines qui entretiendraient l'inflammation et l'immunodépression. Ces PRRs jouent aussi un rôle fondamental dans l'homéostasie et la réparation du parenchyme pulmonaire. Il a été démontré qu'une stimulation excessive et/ou chronique des PRRs par des molécules types DAMPs peut entraîner un remodelage pathologique, voire une destruction du parenchyme pulmonaire.

Trois PPRs sont principalement impliqués dans le VILI : les Toll like receptors (TLR4, TLR3, TLR2) [30-32], NLRP3 (inflammasome/IL-1 $\beta$ ) [33-34] et le receptor for advanced glycation end products (RAGE) [35]. La plupart des études qui ont démontré l'importance de ces récepteurs 


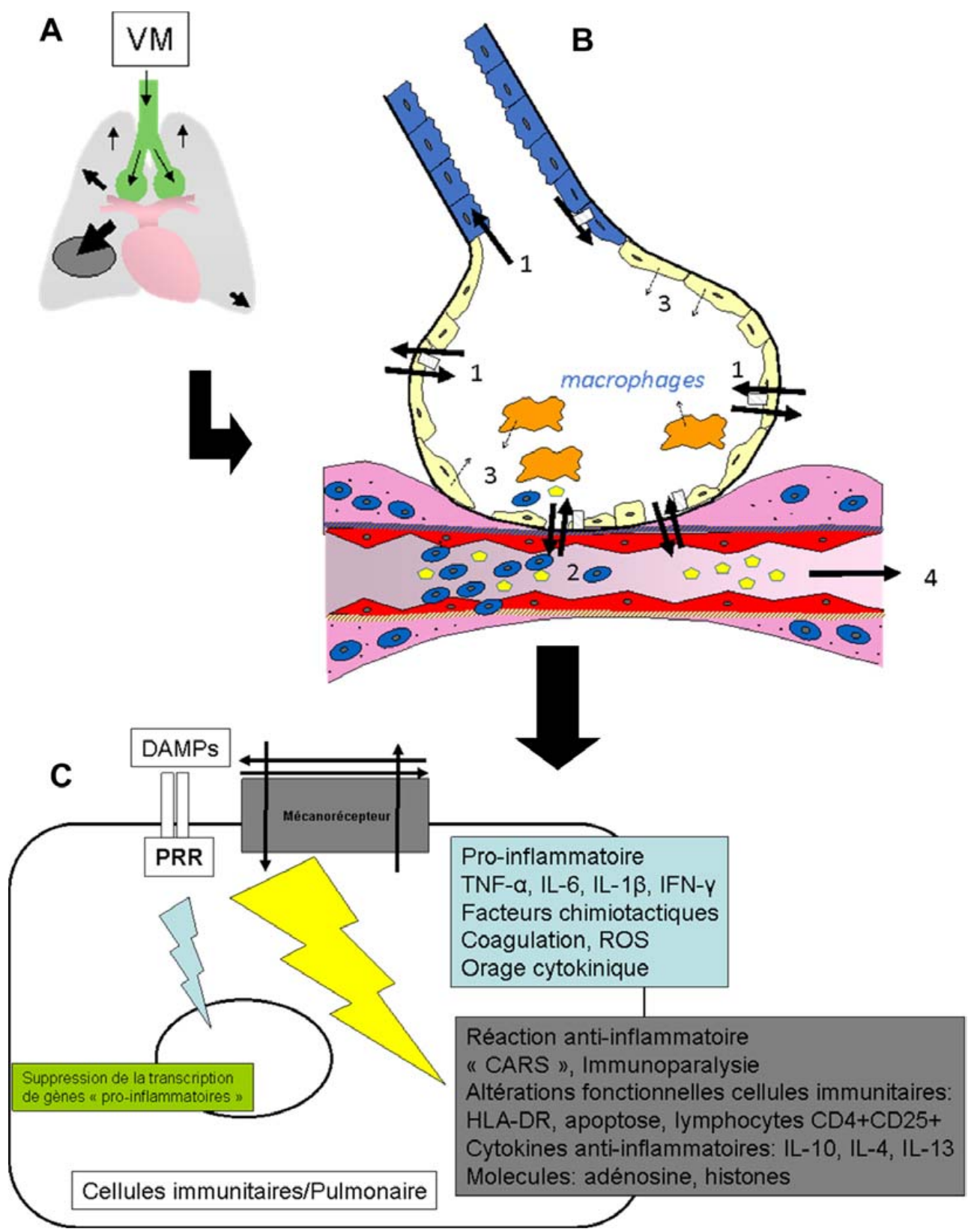

Fig. 1 Représentation schématique du biotraumatisme et de la dysfonction immunitaire induite par la ventilation mécanique. A : la ventilation en pression positive va générer des forces mécaniques qui ne seront pas réparties de façon homogène si le poumon est lésé. B : 1) dans l'alvéole, ces forces mécaniques vont être traduites en signaux biologiques par les cellules grâce aux mécanorécepteurs (mécanotransduction) ; 2) parallèlement, les lésions de la barrière alvéolo-capillaire préexistantes sont majorées par les conséquences de la mécanotransduction, entraînant un afflux de polynucléaires neutrophiles (PNN) et de damage-associated molecular pattern molecules (DAMPs) ou alarmines ; 3) une réaction inflammatoire impliquant essentiellement les PNN, les macrophages et les cellules épithéliales va entraîner l'activation de ces cellules, une diminution des défenses immunitaires locales, aboutissant au 4) passage systémique de cette réaction initialement locale. $\mathrm{C}$ : dans la cellule, l'interaction alarmine- Pattern recognition receptors (PRRs) ou l'activation de mécanorécepteur aboutira parallèlement à une réaction pro- et anti-inflammatoire entraînant une immunodépression. CARS : compensatory anti-inflammatory response syndrome ; IFN- $\gamma$ : interféron- $\gamma$; IL : interleukine ; ROS : espèces réactives de l'oxygène ; TNF- $\alpha$ : Tumor necrosis factor- $\alpha$

dans la dysfonction immunitaire induite par la VM ont utilisé des modèles animaux de VILI en comparant animaux de type sauvage à des animaux dont les gènes ont été délétés pour un de ces récepteurs.
Les principales molécules pouvant jouer un rôle de DAMPs dans le VILI sont : l'acide hyaluronique et d'autres composants de la matrice extracellulaire, Heat shock protein, High-mobility box group-1 (HMGB1), la protéine S100, 
l'inhibiteur de l'activateur du plasminogène (PAI)-1, l'adénosine-5'-triphosphate et l'acide urique [32,36].

Les principales voies impliquées sont les suivantes : les TLR activent une voie de signalisation menant à l'activation des facteurs de transcription nuclear factor-kappa $B(\mathrm{NF}-\mathrm{kB})$ et AP1, qui régulent l'expression inductible des cytokines inflammatoires comme le TNF $\alpha$, l'IL-1 ou l'IL-6, et des molécules costimulatrices CD80 et CD86. Le rôle de NLRP3 et de l'inflammasome dans l'inflammation lors du VILI est lié à l'IL1- $\beta$. RAGE pourrait activer la nicotinamide adenine dinucleotide phosphate-oxidase (NADPH), oxydase des PNN, et jouer ainsi un rôle dans les lésions induites par la production d'espèces réactives de l'oxygène par les PNN [30-36].

\section{Exemples d'anomalies induites par la ventilation mécanique : HMGB1}

L'étude du rôle de High-mobility group protein B1 (HMGB1) dans la dysfonction immunitaire induite par la VM illustre parfaitement les interactions PRRs-alarmines développées précédemment.

HMGB1 a initialement été décrit comme une protéine nucléaire non histone de liaison à l'acide désoxyribonucléique $(\mathrm{ADN})$ [37]. Il a par la suite été démontré que HMGB1 peut être libérée par les cellules apoptotiques ainsi que par les macrophages activés et d'autres populations cellulaires [38-40]. Les taux plasmatiques d'HMGB1 sont élevés dans l'inflammation aiguë, le choc septique, les brûlures étendues, le choc hémorragique, ainsi que dans les atteintes pulmonaires inflammatoires comme le SDRA [37,41-45]. HMGB1 participe à la potentialisation de l'activité proinflammatoire de médiateurs et cytokines ainsi que du LPS. Enfin, les thérapies dirigées contre HMGB1 ont montré un bénéfice dans la résolution de pathologies inflammatoires aiguës, des lésions pulmonaires, du sepsis ou des lésions d'ischémie-reperfusion [44-46]. L'utilisation d'un anticorps spécifique dirigé contre HMGB1 a permis de diminuer les lésions observées de VILI [44]. Le rôle d'HMGB1 comme alarmine dans le contexte de dysfonction immunitaire induite par la VM est fonction du récepteur (PRR) impliqué :

- en se liant au récepteur RAGE dans un contexte infectieux : l'activation des PNN, associée à la production de peptides antibactériens, de ROS, de cytokines et d'autres médiateurs de l'inflammation, ainsi que la libération de NETs (produits par la libération de l'ADN dans le milieu extracellulaire par les PNN), jouent un rôle central dans la défense innée et la modulation de l'inflammation [47,48]. La capacité des NETs à « piéger » et détruire les bactéries endovasculaires a été démontrée, limitant ainsi la diffusion microbienne [47]. En plus de l'ADN, les NET contiennent d'autres protéines qui peuvent faciliter l'éradication microbienne (his- tones, élastase et la myélopéroxydase) [49]. Les NET sont formés en réponse à des stimuli pro-inflammatoires, comme le LPS, l'IL- 8 et le TNF- $\alpha$, mais aussi par la stimulation bactérienne directe. Le mécanisme principal de leur formation fait intervenir la production de ROS via la stimulation de la NADPH oxydase [47-49]. Une diminution de production de ROS par les PNN sera donc responsable d'une diminution de leur activité bactéricide par deux mécanismes $[49,50]$ et entraînera une immunodépression. HMGB1 en se liant au récepteur RAGE entraîne une diminution de production de ROS par les PNN et donc une diminution de leur bactéricidie ;

- en se liant au TLR4 dans un contexte d'inflammation stérile, nous avons démontré que HMGB1 pouvait induire la formation de NETs et majorer les lésions pulmonaires [46]. Il a été démontré que le taux plasmatique d'HMGB1 avait une valeur pronostique, et surtout que la capacité des patients à produire des anticorps dirigés contre HMGB1 avait une influence sur la survie [51]. En effet, des autoanticorps dirigés contre HMGB1 pouvaient apparaître après un choc septique et étaient associés à une amélioration du pronostic de ces patients.

\section{Conséquences cliniques. Stratégies préventive et thérapeutique}

\section{Conséquences cliniques}

L'extension systémique des conséquences d'une réaction inflammatoire initialement locale va entrainer une surmorbidité et une surmortalité du patient en réanimation. En effet, les lésions de la barrière alvéolocapillaire permettent la diffusion de médiateurs dans la circulation systémique [52] :

- la réaction inflammatoire engendrée ou aggravée par la VM pourrait être une des causes du développement d'une défaillance multiviscérale chez les patients de réanimation. Plusieurs études cliniques ont démontré que l'application d'un $\mathrm{Vt}>10 \mathrm{~mL} / \mathrm{kg}$ de poids idéal entraînait une augmentation des concentrations plasmatiques de cytokines pro-inflammatoires, et que la réduction du $\mathrm{Vt}$ permettait de les décroître $[6,14,19]$. Ces données pourraient expliquer la surmortalité observée lors de l'essai clinique de l'ARDS Network dans le groupe haut $\mathrm{Vt}$, non expliquée par la survenue plus fréquente de barotraumatisme ou une dégradation du rapport $\mathrm{PaO}_{2} / \mathrm{FiO}_{2}$ [7]. Plusieurs études ont démontré que le VILI était associé à une dysfonction d'organes, incluant l'insuffisance rénale aiguë et les lésions intestinales [53];

- immunodépression : la généralisation du syndrome inflammatoire va entrainer un CARS, qui contribue à l'immunodépression des patients en réanimation et donc à la 
survenue d'infections nosocomiales. Il n'est pas certain qu'on puisse lui imputer en totalité la survenue de pneumopathies virales. Cependant, la survenue d'une réelle immunodépression systémique favorise de telles réactivations virales [54,55]. Dans plusieurs études, animales pour la plupart, a été constatée une diminution claire de la réponse à l'infection des poumons ventilés [56];

- les lésions induites entraînent des translocations bactériennes (poumon vers le sang), diminuent la réponse inflammatoire lors d'une exposition aux bactéries [57].

\section{Stratégies préventive et thérapeutique}

Il n'y a pas de traitement des modifications immunologiques induites par la VM. Cependant, toutes les mesures qui diminuent la durée de VM ou les lésions induites par celle-ci participent à une diminution de la morbidité et de la mortalité des patients ventilés comme le décubitus ventral, l'utilisation des curares, le recrutement alvéolaire ou l'utilisation de l'extracorporeal membrane oxygenation (ECMO) [6]. La diminution du Vt et le monitorage de la pression de plateau a permis de diminuer la mortalité des patients au cours du SDRA. L'émergence de biomarqueurs et le développement d'anticorps spécifiques contre ces molécules pourrait constituer une voie thérapeutique prometteuse.

Enfin, il semblerait que la VM en elle-même puisse induire des lésions, même si celle-ci est mise en place lors d'une anesthésie générale ou en utilisant des $\mathrm{Vt}<12 \mathrm{ml} / \mathrm{kg}$. En effet, dans un travail récent, il a été démontré que l'utilisation peropératoire d'une ventilation protectrice lors d'une chirurgie abdominale, permettait de limiter la diminution de compliance pulmonaire en fin d'intervention, mais surtout diminuait significativement les complications infectieuses postopératoires (pulmonaires et non pulmonaires) [58]. Ceci pourrait être la traduction clinique de données expérimentales. En effet, il a été montré chez l'homme que la VM induisait rapidement une immunodépression, même pour des durées très courtes de VM $[59,60]$.

\section{Conclusion}

Les conséquences immunologiques de la VM chez le patient de réanimation sont comparables à celles décrites lors du sepsis, induisant une immunodépression $[6,61]$. Une approche thérapeutique pourrait donc bénéficier des mêmes stratégies, la différence fondamentale étant que le début de l'agression par la VM est parfaitement connu, renforçant ainsi l'efficacité des mesures préventives. L'effet bénéfique sur la mortalité et la morbidité constaté avec toutes les stratégies permettant de réduire les durées de VM et les lésions de surdistension trouvent en partie une explication dans la prévention des phénomènes biologiques décrits dans le contexte de biotraumatisme. À l'avenir, le développement d'anticorps spécifiques contre certaines alarmines apportera peut-être une voie thérapeutique intéressante.

Conflit d'intérêt : J.M. Tadié, A. Gacouin et Y. Le Tulzo déclarent ne pas avoir de conflit d'intérêt.

\section{Références}

1. Dreyfuss D, Saumon G (1998) Ventilator-induced lung injury: lessons from experimental studies. Am J Respir Crit Care Med 15:294-323

2. Vlahakis NE, Hubmayr RD (2005) Cellular stress failure in ventilator-injured lungs. Am J Respir Crit Care Med 171:1328-42

3. Jaecklin T, Otulakowski G, Kavanagh BP (2010) Do soluble mediators cause ventilator-induced lung injury and multi-organ failure? Intensive Care Med 36:750-7

4. Hotchkiss RS, Monneret G, Payen D (2013) Sepsis-induced immunosuppression: from cellular dysfunctions to immunotherapy. Nat Rev Immunol 13:862-74

5. Vincent JL, Bihari DJ, Suter PM, et al (1995) The prevalence of nosocomial infection in intensive care units in Europe. Results of the European Prevalence of Infection in Intensive Care (EPIC) Study. EPIC International Advisory Committee. JAMA 274:639-44

6. Slutsky AS, Ranieri VM (2013) Ventilator-induced lung injury. N Engl J Med 369:2126-36

7. The Acute Respiratory Distress Syndrome Network (2000) Ventilation with lower tidal volumes as compared with traditional tidal volumes for acute lung injury and the acute respiratory distress syndrome. N Engl J Med 342:1301-8

8. Mercat A, Richard JC, Vielle B, et al (2008). Positive endexpiratory pressure setting in adults with acute lung injury and acute respiratory distress syndrome: a randomized controlled trial. JAMA 299:646-55

9. Gattinoni L, Pesenti A, Avalli L, et al (1987) Pressure-volume curve of total respiratory system in acute respiratory failure. Computed tomographic scan study. Am Rev Respir Dis 136:730-6

10. Jacob AM, Gaver DP 3rd (2012) Atelectrauma disrupts pulmonary epithelial barrier integrity and alters the distribution of tight junction proteins ZO-1 and claudin 4. J Appl Physiol 113:1377-87

11. Slutsky AS (1999) Lung injury caused by mechanical ventilation. Chest 116:9S-15S

12. Pugin J (2003) Molecular mechanisms of lung cell activation induced by cyclic stretch. Crit Care Med 31:S200-6

13. Liu M, Tanswell AK, Post M (1999) Mechanical force-induced signal transduction in lung cells. Am. J. Physiol 277:L667-83

14. dos Santos CC, Slutsky AS (2006) The contribution of biophysical lung injury to the development of biotrauma. Annu Rev Physiol 68:585-618

15. Chapman KE, Sinclair SE, Zhuang D, et al (2008) Cyclic mechanical strain increases reactive oxygen species production in pulmonary epithelial cells. Am J Physiol Lung Cell Mol Physiol 38:362-70

16. Pugin J, Dunn-Siegrist I, Dufour J, et al (2008) Cyclic stretch of human lung cells induces an acidification and promotes bacterial growth. Am J Respir Cell Mol Biol 38:362-70

17. Janmey PA (1998) The cytoskeleton and cell signaling: component localization and mechanical coupling. Physiol Rev 78:763-81

18. Zupancich E, Paparella D, Turani F, et al (2005) Mechanical ventilation affects inflammatory mediators in patients undergoing cardiopulmonary bypass for cardiac surgery: a randomized clinical trial. J Thorac Cardiovasc Surg 130:378-83 
19. Emr B, Gatto LA, Roy S, et al (2013) Airway pressure release ventilation prevents ventilator-induced lung injury in normal lungs. JAMA Surg 148:1005-12

20. Pugin J, Dunn I, Jolliet P, et al (1998) Activation of human macrophages by mechanical ventilation in vitro. Am J Physiol 275:L1040-50

21. Tremblay LN, Miatto D, Hamid Q, et al (2002) Injurious ventilation induces widespread pulmonary epithelial expression of tumor necrosis factor- $\alpha$ and interleukin-6 messenger RNA. Crit Care Med 30:1693-700

22. Martin TR, Frevert CW (2005) Innate immunity in the lungs. Proc Am Thorac Soc 2:403-11

23. Opitz B, van Laak V, Eitel J, et al (2010) Innate immune recognition in infectious and noninfectious diseases of the lung. Am J Respir Crit Care Med 181:1294-309

24. Chastre J, Fagon JY (2002) Ventilator-associated pneumonia. Am J Respir Crit Care Med 165:867-903

25. Grommes J, Soehnlein O (2011) Contribution of neutrophils to acute lung injury. Mol Med 17:293-307

26. Belperio JA, Keane MP, Burdick MD, et al (2002) Critical role for CXCR2 and CXCR2 ligands during the pathogenesis of ventilator-induced lung injury. J Clin Invest 110:1703-16

27. Ravichandran K S, Lorenz U (2007) Engulfment of apoptotic cells: signals for a good meal. Nat Rev Immunol 7:964-74

28. Caudrillier A, Kessenbrock K, Gilliss BM, et al (2012) Platelets induce neutrophil extracellular traps in transfusion-related acute lung injury. J Clin Invest 122:2661-71

29. Bianchi ME (2007) DAMPs, PAMPs and alarmins: all we need to know about danger. J Leukoc Biol 81:1-5

30. Vaneker M, Joosten LA, Heunks LM, et al (2008) Lowtidal-volume mechanical ventilation induces a toll-like receptor 4-dependent inflammatory response in healthy mice. Anesthesiology 109:465-72

31. Murray LA, Knight DA, McAlonan L, et al (2008) Deleterious role of TLR3 during hyperoxia-induced acute lung injury. Am J Respir Crit Care Med 178:1227-37

32. Kuipers MT, van der Poll T, Schultz MJ, et al (2011) Benchto-bedside review: Damage-associated molecular patterns in the onset of ventilator-induced lung injury. Crit Care 15:235

33. Kuipers MT, Aslami H, Janczy JR, et al (2012) Ventilatorinduced lung injury is mediated by the NLRP3 inflammasome. Anesthesiology 116:1104-15

34. Wu J, Yan Z, Schwartz DE (2013) Activation of NLRP3 inflammasome in alveolar macrophages contributes to mechanical stretch-induced lung inflammation and injury. J Immunol 190:3590-9

35. Reynolds PR, Schmidt RE, Kasteler SD, et al (2010) Receptors for advanced glycation end products targeting protects against hyperoxia-induced lung injury in mice. Am J Respir Cell Moll Biol 42:545-51

36. Agrawal A, Zhuo H, Brady S, et al (2012) Pathogenetic and predictive value of biomarkers in patients with ALI and lower severity of illness: results from two clinical trials. Am J Physiol Lung Cell Mol Physiol 303:L634-9

37. Andersson U, Tracey KJ (2011) HMGB1 is a therapeutic target for sterile inflammation and infection. Annu Rev Immunol 29:139-62

38. Chorny A, Anderson P, Gonzalez-Rey E, et al (2008) Ghrelin protects against experimental sepsis by inhibiting high-mobility group box 1 release and by killing bacteria. J Immunol 180:8369-77

39. Tadie JM, Bae HB, Deshane JS, et al (2012) TLR4 engagement inhibits AMPK activation through a HMGB1 dependent mechanism. Mol Med 18:659-68

40. Tsung A, Klune JR, Zhang X, et al (2007) HMGB1 release induced by liver ischemia involves Toll-like receptor 4 dependent reactive oxygen species production and calcium-mediated signaling. J Exp Med 204:2913-23
41. Kim JY, Park JS, Strassheim D, et al (2005). HMGB1 contributes to the development of acute lung injury after hemorrhage. Am J Physiol Lung Cell Mol Physiol 288:L958-L965

42. Friggeri A, Yang Y, Banerjee S, et al (2010) HMGB1 inhibits macrophage activity in efferocytosis through binding to the avß3-integrin. Am J Physiol Cell Physiol 299:C1267-C1276

43. Sha Y, Zmijewski J, Xu Z, et al (2008) HMGB1 develops enhanced proinflammatory activity by binding to cytokines. J Immunol 180:2531-7

44. Ogawa EN, Ishizaka A, Tasaka S, et al (2006) Contribution of high-mobility group box-1 to the development of ventilatorinduced lung injury. Am J Respir Crit Care Med 174: 400-7

45. Shimazaki J, Matsumoto N, Ogura H, et al (2012) Systemic involvement of HMGB1 and therapeutic effect of anti-HMGB1 antibody in a rat model of crush injury. Shock 37: 634-638

46. Tadie JM, Bae HB, Jiang S, et al (2013) HMGB1 can induce neutrophils extracellular traps through TLR4. Am J Physiol Lung Cell Mol Physiol 304:L342-9

47. Clark SR, Ma AC, Tavener SA, et al (2007) Platelet TLR4 activates neutrophil extracellular traps to ensnare bacteria in septic blood. Nat Med 13:463-9

48. Brinkmann V, Reichard U, Goosmann C, et al (2004) Neutrophil extracellular traps kill bacteria. Science 303:1532-5

49. Mantovani A, Cassatella MA, Costantini C, et al (2011) Neutrophils in the activation and regulation of innate and adaptive immunity. Nat Rev Immunol 11:519-31

50. Tadié JM, Bae HB, Banerjee S, et al (2012) Differential activation of RAGE by HMGB1 modulates neutrophil-associated NADPH oxidase activity and bacterial killing. Am J Physiol Cell Physiol 302:C249-56

51. Barnay-Verdier S, Fattoum L, Borde C, et al (2011) Emergence of autoantibodies to HMGB1 is associated with survival in patients with septic shock. Intensive Care Med 37:957-62

52. Haitsma JJ, Uhlig S, Goggel R, et al (2000) Ventilator-induced lung injury leads to loss of alveolar and systemic compartmentalization of tumor necrosis factor- $\alpha$. Intensive Care Med 26:1515-22

53. Imai Y, Parodo J, Kajikawa O, et al (2003) Injurious mechanical ventilation and end-organ epithelial cell apoptosis and organ dysfunction in an experimental model of acute respiratory distress syndrome. JAMA 289:2104-12

54. Limaye AP, Kirby KA, Rubenfeld GD, et al (2008) Cytomegalovirus reactivation in critically ill immunocompetent patients. JAMA 300:413-22

55. Luyt CE, Combes A, Deback C, et al (2007) Herpes simplex virus lung infection in patients undergoing prolonged mechanical ventilation. Am J Respir Crit Care Med 175:935-42

56. Verbrugge SJ, Sorm V, van 't Veen A, et al (1998) Lung overinflation without positive end-expiratory pressure promotes bacteremia after experimental Klebsiella pneumoniae inoculation. Intensive Care Med 24:172-7

57. Lin CY, Zhang H, Cheng KC, et al (2003) Mechanical ventilation may increase susceptibility to the development of bacteremia. Crit Care Med 31:1429-34

58. Futier E, Constantin JM, Paugam-Burtz C, et al (2013) A trial of intraoperative low-tidal-volume ventilation in abdominal surgery. N Engl J Med 369:428-37

59. Emr B, Gatto LA, Roy S, et al (2013) Airway pressure release ventilation prevents ventilator-induced lung injury in normal lungs. JAMA Surg 148:1005-12

60. Plötz FB, Vreugdenhil HA, Slutsky AS, et al (2002) Mechanical ventilation alters the immune response in children without lung pathology. Intensive Care Med 28:486-92

61. Le Tulzo Y, Pangault C, Amiot L, et al (2004) Monocyte human leukocyte antigen-DR transcriptional downregulation by cortisol during septic shock. Am J Respir Crit Care Med 169:1144-51 